Europhysics Letters

PREPRINT

\title{
Fluctuating lattice Boltzmann
}

\author{
${ }^{1}$ R. Adhikari, ${ }^{2}$ K. Stratford, ${ }^{1}$ M.E. Cates and ${ }^{3}$ A.J. Wagner \\ ${ }^{1}$ School of Physics and ${ }^{2}$ EPCC, University of Edinburgh, JCMB Kings Buildings, May- \\ field Road, Edinburgh EH9 3JZ GB; ${ }^{3}$ Dept of Physics, North Dakota State University, \\ Fargo ND58105, USA
}

\begin{abstract}
The lattice Boltzmann algorithm efficiently simulates the Navier Stokes equation of isothermal fluid flow, but ignores thermal fluctuations of the fluid, important in mesoscopic flows. We show how to adapt the algorithm to include noise, satisfying a fluctuation-dissipation theorem (FDT) directly at lattice level: this gives correct fluctuations for mass and momentum densities, and for stresses, at all wavevectors $k$. Unlike previous work, which recovers FDT only as $k \rightarrow 0$, our algorithm offers full statistical mechanical consistency in mesoscale simulations of, e.g., fluctuating colloidal hydrodynamics.
\end{abstract}

The lattice Boltzmann equation (LBE) is a widely used lattice formulation of fluid mechanics [1]. It offers a faithful discretization of the Navier Stokes equation of isothermal, incompressible fluid flow, and is very well adapted to parallel computation [2]. While used for large-scale fluid dynamics simulations such as flows around aircraft [3], the LBE approach is particularly adapted to simulating mesoscopic problems [4]. These include, e.g., porous medium flows and flows of complex and multicomponent fluids with microstructure [5-8]. The latter can be modelled using various extensions of the basic algorithm for a single component fluid as considered here [8-10].

However, the Navier Stokes equation, and with it the LBE, ignores thermal fluctuations. While these may safely be ignored in macroscopic fluid-dynamical flows, at mesoscopic length scales they form an essential part of the physics [11]. This applies even in linear problems such as the Brownian motion of a colloidal particle suspended in a simple fluid: if that fluid is simulated using the LBE, no Brownian motion occurs [8]. Fluctuations are also central to nonlinear phenomena such as mode-coupling effects and long-time tails [12]. By the same token, extensions of the LBE to fluid mixtures [9] and amphiphilic solutions [10] cannot address critical phenomena, where fluctuations dominate.

In this letter we present a fluctuating LBE (FLBE). This offers a fully consistent discretization of the equations of fluctuating nonlinear hydrodynamics for an isothermal fluid, opening the way to more accurate and efficient simulation of many of the mesoscale physics problems mentioned previously, such as colloid hydrodynamics. Its generalization to multicomponent fluids is conceptually straightforward; we pursue this elsewhere [13]. Our work also raises broader issues for numerical statistical mechanics: how best to implement fluctuationdissipation theorems (FDTs), derived in the continuum with respect for appropriate conservation laws, in a system discretized in space and time [14]. We contend that accuracy and efficiency are best combined if FDT is made to hold directly on the discretized dynamics.

(C) EDP Sciences 
We start with the LBE for a single-component fluid, which can be viewed as a discretization of the Boltzmann equation for the collisional dynamics of a dilute gas [15]:

$$
f_{i}\left(\mathbf{x}+\mathbf{c}_{i}, t+1\right)=f_{i}(\mathbf{x}, t)+\mathcal{L}_{i j}\left(f_{j}(\mathbf{x}, t)-f_{j}^{0}(\mathbf{x}, t)\right)
$$

Here $f_{i}$ represents the local mass density of particles in a phase space cell $\left(\mathbf{x}, \mathbf{c}_{i}\right)$, and is normalized so that $\sum_{i=1}^{n} f_{i}=\rho(\mathbf{x})$, the fluid mass density at $\mathbf{x}$. The spatial coordinates $\mathbf{x}$ are discretized onto a unit lattice; a finite set of $n$ velocities $\mathbf{c}_{i}$ is chosen so that in timestep $\Delta t=1$, the resulting 'streaming' displacements $\Delta \mathbf{x}_{i}=\mathbf{c}_{i} \Delta t$ are lattice vectors. The local momentum density and flux are $g_{\alpha}=\sum_{i} f_{i} c_{i \alpha}$ and $\Pi_{\alpha \beta}=\sum_{i} f_{i} c_{i \alpha} c_{i \beta}$; Greek indices indicate Cartesian directions. The equilibrium distribution $f_{i}^{0}$ is conditioned by the local values of $\rho$, $g_{\alpha}$ and $\Pi_{\alpha \beta}$. The collision operator $\mathcal{L}_{i j}$ linearly relaxes the local phase-cell densities towards this equilibrium. For well chosen lattices and collision operators, the LBE is known to recover the isothermal Navier-Stokes equation in the continuum limit at low Mach number [1].

The $f_{i}$ in Eq11 are ensemble-averaged local distribution functions. Although these depend on temperature (indeed, $f_{i}^{0}$ for a fluid at rest is Boltzmann distributed [16]), they describe only the mean densities in each phase space cell. Accordingly, they neglect the fluctuating stress $s_{\alpha \beta}$ in the equations of fluctuating nonlinear hydrodynamics [19] for an isothermal fluid:

$$
\begin{gathered}
\partial_{t} \rho+\nabla_{\alpha} g_{\alpha}=0 \\
\partial_{t} g_{\alpha}+\nabla_{\beta} \Pi_{\alpha \beta}=0 \\
\Pi_{\alpha \beta}=g_{\alpha} v_{\beta}+p \delta_{\alpha \beta}+\eta_{\alpha \beta \gamma \delta} \nabla_{\gamma} v_{\delta}+s_{\alpha \beta} .
\end{gathered}
$$

In Eqs $2 v_{\alpha}=g_{\alpha} / \rho$ is the local fluid velocity, $p$ is the pressure in a quiescent fluid (given here by an ideal gas equation of state $p=\rho c_{s}^{2}$, with $c_{s}$ the isothermal sound speed), and $\eta_{\alpha \beta \gamma \delta}$ is a tensor of viscosities. The fluctuating stress $s_{\alpha \beta}$ is a zero-mean Gaussian random variable whose variance, for a fluid at temperature $T$, is fixed by the fluctuation-dissipation theorem (FDT) to be $\left\langle s_{\alpha \beta}(\mathbf{x}, t) s_{\gamma \delta}\left(\mathbf{x}^{\prime}, t^{\prime}\right)\right\rangle=2 k_{B} T \eta_{\alpha \beta \gamma \delta} \delta\left(\mathbf{x}-\mathbf{x}^{\prime}\right) \delta\left(t-t^{\prime}\right)$.

An important precursor to our work is that of Ladd [8]. His method consists of adding a stochastic piece to the microscopic stress tensor in Eq11. Following through to the continuum limit, Eq2 is recovered. However, this process ensures only that FDT holds in the hydrodynamic limit $k \rightarrow 0$. In practice, no numerical algorithm is ever used in this limit, which would require infinite computational resources. Indeed, in the noiseless LBE, acceptable hydrodynamic behavior of the fluid is maintained right up to $k \simeq 2$ [20]; accordingly accurate colloid hydrodynamics is achieved with colloids of rather small radius, e.g., $R=2.5$ [8]. (Lengths are expressed in lattice units, so that $k=2$ has a wavelength of $\pi$ ). But unless the correct noise behavior is implemented over a similarly wide $k$ range, breakdown of FDT at high $k$ is liable to infect the whole simulation, once noise is added. We show below, for a simple benchmark problem of colloids in traps, that this can indeed occur, resulting in errors of order $10 \%$. (These errors might be reduced by making $R$ several times larger at fixed volume fraction; but this is not computationally efficient.) Moreover, as found below, such errors show nontrivial dependence on parameters such as the colloid volume fraction. Without exhaustive testing, therefore, one cannot know whether Ladd's algorithm is accurate or not, in any given region of parameter space. In contrast, our own algorithm appears to give good equilibration of colloidal degrees of freedom whenever the suspending fluid is accurately in equilibrium.

To make progress, we note that in addition to the hydrodynamic degrees of freedom appearing in Eq2 the LBE necessarily involves the dynamics of local, non-hydrodynamic modes often called 'ghosts' [21,22]. These are needed in Eq11 to maintain isotropic and Gallilean 
${ }^{1}$ R. Adhikari, ${ }^{2}$ K. Stratford, ${ }^{1}$ M.E. Cates and ${ }^{3}$ A.J. Wagner: Fluctuating lattice Boltzmann3

invariant hydrodynamics [15,23]. As shown below, the method of Ref. [8] effectively sets $T=0$ for the ghost mode noise. There is then a risk that ghosts drain thermal fluctuations away from the hydrodynamic modes, which therefore may never reach equilibrium.

To create our improved FLBE, we promote Eq廿into a discrete Langevin equation, where the $f_{i}$ are interpreted as instantaneous, fluctuating densities in phase space:

$$
f_{i}\left(\mathbf{x}+\mathbf{c}_{i}, t+1\right)=f_{i}(\mathbf{x}, t)+\mathcal{L}_{i j}\left(f_{i}(\mathbf{x}, t)-f_{i}^{0}(\mathbf{x}, t)\right)+\xi_{i}
$$

with noise terms $\xi_{i}(\mathbf{x}, t)$ that give fluctuations in the populations in each phase space cell. To recover thermal equilibrium, the $\xi_{i}$ must be linked, by an FDT, to all sources of dissipation in the collision operator $\mathcal{L}_{i j}$. The required FDT must allow that, at a given site and timestep, the $\xi_{i}$ are correlated in such a way as to exactly conserve $\rho$ and $g_{\alpha}$. To recover continuum hydrodynamics, the collision process necessarily avoids relaxing the conserved quantities, which propagate only via the 'streaming step' $\mathbf{x} \rightarrow \mathbf{x}+\mathbf{c}_{i}$ in Eq[1]. Below we diagonalize the collisional dissipation, using established methods [23]. In contrast to previous work, we determine the accompanying noise structure consistent with statistical mechanical principles.

First, note that each unnormalised eigenvector $m_{i}^{a}$ of $\mathcal{L}_{i j}$ with eigenvalue $\left(-1 / \tau_{a}\right)$ is associated with a corresponding local density $M^{a}(\mathbf{x}, t)$ via

$$
M^{a}(\mathbf{x}, t)=\sum_{i=1}^{n} m_{i}^{a} f_{i}(\mathbf{x}, t), a=1 \ldots n
$$

For example, the density $\rho(\mathbf{x}, t)$ equates to $M^{1}(\mathbf{x}, t)$ where $m_{i}^{1}=1 \forall i$. For a general LBE in $d$ dimensions containing $n$ velocities (a ' $\mathrm{D} d \mathrm{Q} n$ model'), there are precisely $n$ eigenvectors, corresponding to the $n$ degrees of freedom contained in the $f_{i}$ at a given site. Each $M^{a}$ relaxes at a rate given by the eigenvalue $-1 / \tau_{a}$. Conserved hydrodynamic variables (mass, momentum, but not stress) do not relax; we set the corresponding eigenvalues to zero $\left(-1 / \tau_{a}=\right.$ 0 ) without loss of generality. Note that our method allows for a multi-relaxation time kernel but does not assume it; ghost noise is important even with a single relaxation time.

A complete mode count then consists of the null eigenvector $m_{i}^{1}$ corresponding to the density $\rho$; the $d$ null eigenvectors $c_{i \alpha}$ corresponding to the $d$ components of the momentum $g_{\alpha} ; \frac{1}{2} d(d+1)$ eigenvectors $Q_{i \alpha \beta}=c_{i \alpha} c_{i \beta}-c_{s}^{2} \delta_{\alpha \beta}$ corresponding to (independent components of) the deviatoric momentum flux $S_{\alpha \beta}=\Pi_{\alpha \beta}-\nu k_{B} T \delta_{\alpha \beta}$, where $\nu$ is the number density; and the remaining $n-\left(1+d+\frac{1}{2} d(d+1)\right)$ ghost mode eigenvectors. The latter are modeldependent, but readily computed from $\mathcal{L}_{i j}$ in any given implementation of the LBE [1]. Our $n$ eigenvectors form a complete, orthogonal basis in the finite velocity space of the LBE. Thus

$$
f_{i}(\mathbf{x}, t)=\sum_{a} w_{i} m_{i}^{a} M^{a}(\mathbf{x}, t) N^{a}
$$

with normalizers $N^{a}$ obeying $N^{a} \sum_{i} w_{i} m_{i}^{a} m_{i}^{b}=\delta_{a b}$ and $w_{i}$ a set of known weights [1].

A similar expansion can be applied to any function defined on the velocity space, including the noise $\xi_{i}(\mathbf{x}, t)$. Let us first set $\xi_{i}=\xi_{i}^{H}+\xi_{i}^{G}$ with $H$ the hydrodynamic subspace and $G$ is its complement, the ghost subspace. Identifying the $H$ eigenvectors as above, we write

$$
\xi_{i}^{H}=\sum_{a \in H} w_{i} m_{i}^{a} \hat{\xi}^{a}(\mathbf{x}, t) N^{a}=w_{i}\left(m_{i}^{1} \hat{\rho}+\frac{c_{i \alpha} \hat{g}_{\alpha}}{c_{s}^{2}}+\frac{Q_{i \alpha \beta} \hat{S}_{\alpha \beta}}{2 c_{s}^{4}}\right)
$$

where the noise terms $\hat{\xi}^{a}$ associated with $\rho, g_{\alpha}, S_{\alpha \beta}$ are denoted by $\hat{\rho} \ldots ;$ repeated Greek indices are summed on. 
Within this framework, conservation laws have a very simple effect: mass and momentum conservation demand $\hat{\rho}=0$ and $\hat{g}_{\alpha}=0$ in Eq[6]. We are left with

$$
\xi_{i}=w_{i} \frac{Q_{i \alpha \beta} \hat{S}_{\alpha \beta}(\mathbf{x}, t)}{2 c_{s}^{4}}+\sum_{g \in G} w_{i} m_{i}^{g} \hat{\xi}^{g}(\mathbf{x}, t) N^{g}
$$

Here the first term is $\xi_{i}^{H}$ : it produces thermal fluctuations in the stress tensor, and is the noise used by Ladd [8]. The remaining terms are $\xi_{i}^{G}$ : these maintain thermal equilibrium for the ghosts, and are new to this work. Our approach differs crucially from earlier work on continuous and discrete stochastic versions of the Boltzmann equation $[17,18]$; these attempted to derive the noise statistics from the collision kernel alone, without taking proper account of mass and momentum conservation.

It remains to determine the covariance matrix of the $n-d-1$ nonzero noises $\left\langle\hat{\xi}^{a} \hat{\xi}^{b}\right\rangle$ that now appear in Eqs 67. Projecting Eq 3 onto the basis of eigenvectors, taking deviations $\delta M^{a}$ around a spatially uniform state and Fourier transforming in space we obtain

$$
\delta M^{a}(\mathbf{k}, t+1)=\Gamma^{a b}(\mathbf{k})\left\{r^{b} \delta M^{b}(\mathbf{k}, t)+\hat{\xi}^{b}(\mathbf{k}, t)\right\}
$$

where $\Gamma^{a b}(\mathbf{k})=\sum_{i=1}^{n} w_{i} m_{i}^{a} m_{i}^{b} N^{b} \exp \left(-i \mathbf{k} \cdot \mathbf{c}_{i}\right)$ and $r^{b}=\left(1-\tau_{b}^{-1}\right)$. Eq 8 represents a set of coupled Langevin equations in discrete time. We now assume that all correlations are $\mathbf{k}$ independent; this is justified below. Next we square both sides of the above equation, average over the noise, and invoke stationarity of equal-time correlators to obtain the required FDT:

$$
\left\langle\hat{\xi}^{a} \hat{\xi}^{b}\right\rangle=\frac{\tau_{a}+\tau_{b}-1}{\tau_{a} \tau_{b}}\left\langle\delta M^{a} \delta M^{b}\right\rangle
$$

The $\tau$-dependence is a standard consequence of the discrete time dynamics in Eq[3 [1,8]. The $d+1$ null modes corresponding to conserved quantities have no dissipation and hence no noise. All remaining modes, including ghosts, have both thermal fluctuations and dissipation; for consistent dynamics, they must have noise. Note from Eq[ 8 that setting $\tau_{b}=1$ for ghosts [8], does not decouple the hydrodynamic modes from the ghost noise, except strictly at $\mathbf{k}=\mathbf{0}$.

To complete our calculation of the noise amplitudes, we now quantify the equilibrium thermal fluctuations $\left\langle\delta M^{a} \delta M^{b}\right\rangle$. This requires a thermodynamic model for our fluctuating fluid. In keeping with the original thinking behind the LBE [1], and also with its practical application (low Mach number), we can choose for this model the thermodynamics of an ideal gas. The fluctuation matrix $\left\langle\delta M^{a} \delta M^{b}\right\rangle$ is then $\mathbf{k}$ independent, as promised above, and computable from knowing that all equilibrium phase-cell occupancies in such a gas obey Poisson statistics [19]. The required matrix $\left\langle\delta M^{a} \delta M^{b}\right\rangle$ then follows by a change of basis from $f_{i}$ to $M^{a}$, using Eq4 In implementing our FLBE numerically, we instead transform in the other direction via Eq[5] This gives from Eq[9] a set of correlated phase-cell noises $\xi_{i}(\mathbf{x}, t)$ for use in Eq3. As shown above, the noises acting on the $n$ different densities $f_{i}$ at site $\mathbf{x}$ are not independent: they derive from only $n-d-1$ underlying independent noises. And, as our eigen-analysis of the collision operator makes clear, they arise from the collisional dissipation of the $\frac{1}{2} d(d+1)$ stress modes and the $\left(n-\left(1+d+\frac{1}{2} d(d+1)\right)\right.$ ghost modes. The computational overhead of adding ghost noise is slight $(\sim 10 \%$ on run time).

The consistency of our FLBE can be assessed by measuring numerically the 'equilibration ratio' (ER) for fluctuating hydrodynamic quantities. This is the ratio of a measured variance to the one required by the Boltzmann distribution [16] at the temperature $T$ chosen for the simulation. We set mass, length, and time units so that $\rho=1$ on an unit lattice. In any 

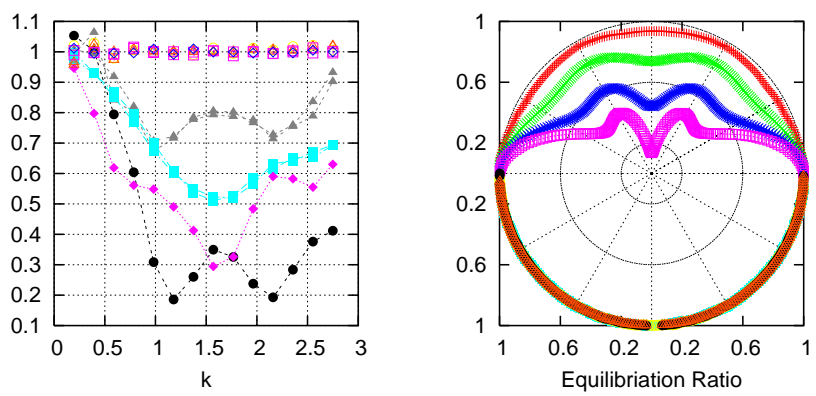

Fig. 1 - Left panel: Equilibration ratios for the Fourier modes of the density (o), momentum $(\triangle)$, and diagonal $(\square)$ and off-diagonal $(\diamond)$ stress components in a D3Q15 model with $\tau=0.75$ shown as a function of $k$ with $\mathbf{k}=k(1,1,1)$. We set $k_{B} T=1 / 3000$. Open symbols: the present work; closed symbols: setting $\xi_{i}^{G}=0$. (Compare Fig.3(d) of [20] where the correct hydrodynamics, without noise, is shown to hold up to $k \sim 2$.) Right panel: $\left\langle\left|g_{x}(\mathbf{k})\right|^{2}\right\rangle$ in a $D 2 Q 9$ model with $\tau=1$, plotted as a function of $\theta$ for various values of $k$, when $\mathbf{k}=k(\cos \theta, \sin \theta)$. The radial coordinate is the equilibriation ratio with unity as the solid circle. Lower half plane: the present work (note that symbols overlap). Upper half plane: setting $\xi_{i}^{G}=0$. Top to bottom at upper right, $k=0.62,1.25,1.88,2.51$.

quiescent fluid the net thermal momentum $\tilde{g}$ in volume $\Delta V$ has variance $\left\langle\tilde{g}^{2}\right\rangle=\Delta V \rho k_{B} T$ [11]. For the ideal gas $k_{B} T=p / \nu=\rho c_{s}^{2} / \nu=c_{s}^{2} / \nu$, where $\nu$ fixes the phase-cell occupancies referred to above, and hence all noise amplitudes. Setting $\Delta V=1$, the on-site thermal velocity $v=\tilde{g} / \rho$ obeys $\left\langle v^{2}\right\rangle=k_{B} T$. Thus, we must have $k_{B} T \ll c_{s}^{2}=1 / 3$ (for $\mathrm{D} d \mathrm{Q} n$ models) to satisfy the low Mach number requirement of the LBE.

We now report results for $D 2 Q 9$ and $D 3 Q 15$ lattices, using a collision operator with unit relaxation time for ghost modes and relaxation time $\tau$ for stress. The viscosity tensor is that of an isotropic fluid, with shear viscosity $\eta=c_{s}^{2}\left(\tau-\frac{1}{2}\right)$ and bulk viscosity $\zeta=\frac{2}{d} \eta$. (This is a particular case of the multirelaxation time operator used in [8].) Fig 1 compares ERs for different hydrodynamic modes on a $D 3 Q 15$ lattice, and shows the dependence on direction in wavevector space of the momentum fluctuations on a $D 2 Q 9$ lattice. We also show the results found by setting $\xi_{i}^{G}=0$ in Eq[7 as per Ref. [8]. As anticipated above, the latter gives acceptable ERs only for $k \ll 1$, with values of $0.2-0.7$ for $1<k<2$, although the noiseless hydrodynamics remains accurate here [20]. In contrast, our algorithm is accurate throughout.

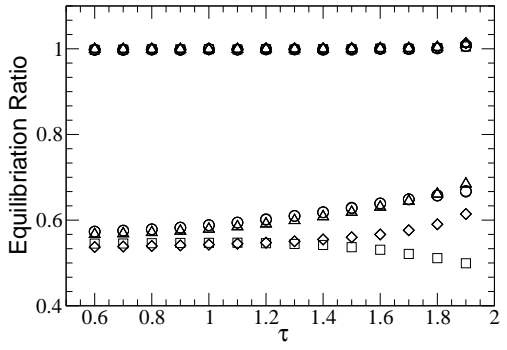

Fig. 2 - Equilibration ratios for on site density $(\square)$, momentum (o) and stress ( $\triangle$ diagonal, $\diamond$ offdiagonal) fluctuations as a function of the relaxation time $\tau$ in a $D 3 Q 15$ model. We set $k_{B} T=1 / 3000$. Upper datasets: the present work (note that symbols overlap). lower datasets: setting $\xi_{i}^{G}=0$. 
EUROPHYSICS LETTERS

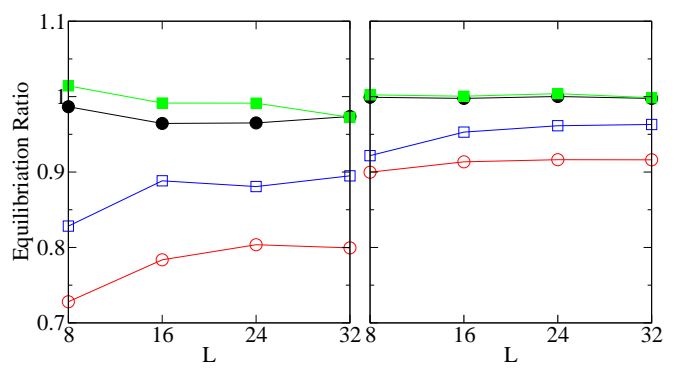

Fig. 3 - Equilibration ratios for colloidal displacements in traps as a function of periodic box size $L$ for $\eta=1 / 6$ (left) $\eta=1 / 60$ (right) and two colloidal radii (circles $R=1.5$, squares $R=2.3$ ) in a $D 3 Q 15$ model. We set $k_{B} T=1 / 19200$. Upper datasets: the present work; lower datasets, the same but setting $\xi_{i}^{G}=0$. Error bars are of order the symbol size.

Figure 2] shows on-site ERs for various fluctuating quantities as a function of $\tau$. These involve integration over all $k$, and could be viewed as a 'worst case' scenario for the local temperature seen by a small colloidal particle. Our algorithm is accurate, even in this limit. We recommend choosing $k_{B} T \leq 1 / 3000$ so that the fluid is accurately incompressible; we then find equilibration at the one percent level or better.

To simulate colloids directly, we have combined our noise implementation with the algorithm of [24] for the colloidal particles [25]. We find results for velocity autocorrelators that offer a slight improvement over the case with ghost noise omitted [26]; but for standard parameter settings both methods give acceptably accurate values for the colloidal diffusion constant $D=k_{B} T /(6 \pi \eta R)$. A more demanding test of equilibration is to simulate colloids in harmonic traps, of the kind often encountered in experiments with optical tweezers [27]. We placed $N$ particles, each in a separate confining potential, at regular intervals on an $L^{3}$ lattice. The particles interact hydrodynamically, but this does not affect the Boltzmann distribution for their thermal displacements within (well-separated) traps.

A statistically decisive comparison can be made for $N=1$, when a given particle is surrounded not by other colloids in independent traps but by its own periodic images, with which it interacts hydrodynamically. In figure 3 we show ERs for the thermal displacement of such a particle. Whereas our results are satisfactory, Ladd's algorithm shows worsening ERs as $L / R$ is decreased [28]. Any attempt to circumvent these large (>10\%) systematic errors arising from omission of ghost noise by defining an 'effective temperature' would merely move those errors into $D$ instead. FDT violations in the ghost sector seemingly present major obstacles to the accurate simulation of colloids, particularly at modest interparticle spacings (modest $L / R$ ) [24] which are overcome by our algorithm [29].

We have also performed tests with $N>1$ (now fixing $L=32$ ). The ER is found for each particle separately by running for $10^{6}$ timesteps with $\eta=1 / 60$ and $k_{B} T=1 / 3000$. (The equilibration time within a trap is of order $r_{0}^{2} / D \sim 10^{4}$.) For $N=64$ particles of $R=2.3$ in traps where the correct rms thermal displacement is $r_{0}=\sqrt{3}$, we find ERs of $0.861 \pm 0.018$ for Ladd's algorithm and $0.957 \pm 0.017$ for our own. For $R=2.3$ and $r_{0}=\sqrt{3} / 2(N=64)$ the results are $0.928 \pm 0.007$ versus our $1.055 \pm 0.011$. For $R=6.23$ and $r_{0}=\sqrt{3} / 2(N=16)$ the results are $0.962 \pm 0.011$ versus our $1.020 \pm 0.015$ [30]. These findings with $N>1$ suggest broadly improved results with our algorithm. However a complete exploration of parameter space would be needed to fully resolve the differences of the two methods: without this, one cannot know in advance when it is safe to neglect ghost noise. This situation is made worse for 
${ }^{1}$ R. Adhikari, ${ }^{2}$ K. Stratford, ${ }^{1}$ M.E. Cates and ${ }^{3}$ A.J. Wagner: Fluctuating lattice Boltzmann7

dense colloids without traps; here the confining potential created on a colloid by its neighbours might not be sampled correctly, but that cannot be checked by explicit calculation.

In conclusion, by writing a discrete Langevin equation at lattice level and carefully applying a fluctuation dissipation theorem, we have derived a fluctuating lattice Boltzmann equation in which the ghost modes are fully thermalized. This removes their fluctuation-draining effects on the hydrodynamic sector at finite $k$. Our method gives improved numerical equilibration of fluctuating quantities at all $k$, thus resolving a potentially major obstacle to the use of lattice Boltzmann methods in the simulation of thermal fluids, including colloid hydrodynamics.

Acknowledgements: We thank Ignacio Pagonabarraga for useful discussions. We thank Tony Ladd for many useful discussions and for showing us his own results on traps [28]. Work funded by EPSRC GR/R67699 (RealityGrid) and GR/S10377.

\section{REFERENCES}

[1] S. Succi, The Lattice Boltzmann Equation for Fluid Dynamics and Beyond (Clarendon Press, Oxford, 2001).

[2] G. Amati, S. Succi, and R. Priva, Int. J. Mod. Phys. C, 8, 869 (1997).

[3] D.P. Lockard, L.S. Luo, S.D. Milder and B.A. Singer, J. Stat. Phys, 107, 423 (2002).

[4] Discrete Modelling and Simulation of Fluid Dynamics, special issue of Phil. Trans. Roy. Soc. A, 360 (2002); P.V. Coveney and S. Succi, eds..

[5] B. Manz, L.F. Gladden, and P.B. Warren, AICHE J 45, 1845 (1999).

[6] V.M. Kendon, et al., Phys. Rev. Lett, 83, 576 (1999); J. Fluid Mech, 440, 147 (2001).

[7] M. Nekovee, P.V. Coveney, H.D. Chen, B.M. Boghosian, Phys. Rev. E, 62, 8282 (2000).

[8] A.J.C. Ladd, J. Fluid Mech, 271, 285 (1994); J. Fluid Mech, 271, 311 (1994).

[9] M.R. Swift, W.R. Osborn, and J.M. Yeomans, Phys. Rev. Lett, 75, 830 (1995).

[10] P.J. Love, et al., Comp. Phys. Comm, 153, 340 (2003).

[11] L.D. Landau and E.M. Lifshitz, Fluid Mechanics (Pergamon Press, Oxford, 1959).

[12] R. Zwanzig, Nonequilibrium Statistical Mechanics (Oxford University Press, 2001).

[13] R. Adhikari et al, work in progress

[14] J.P. Rivet and J.P. Boon, Lattice Gas Hydrodynamics (Cambridge University Press, 2001).

[15] R. Benzi, S. Succi, and M. Vergassola, Phys. Rep. 222, 145 (1992).

[16] 'Boltzmann statistics' here means a polynomial approximation to the Boltzmann distribution.

[17] M. Bixon and R. Zwanzig, Phys. Rev., 187, 267 (1991).

[18] J. W. Dufty and M. H. Ernst, Fields Institute Comm. 6, (1996), e-print comp-gas/93080002.

[19] E.M. Lifshitz and L.P. Pitaevski, Physical Kinetics (Pergamon, Oxford, 1981), Section 19.

[20] O. Behrend, R. Harris, and P.B. Warren, Phys. Rev. E 50, 4586 (1994).

[21] M. Vergassola, R. Benzi, S. Succi, Europhys. Lett 13,411 (1990).

[22] R. Benzi, S. Succi, and M. Vergassola, Europhys. Lett 13,727 (1990).

[23] D. d'Humieres, in Rarefied Gas Dynamics: Theory and Simulations, Prog. Astronaut. Aeronaut, 159450 (1992); D. d'Humieres, in Ref. [4], p.437.

[24] N.-Q. Nguyen, and A.J.C. Ladd, Phys. Rev. E, 66, 046708 (2002).

[25] Our numerics follow Ladd's 'bounce-back' methodology [8,24]. See also [26].

[26] M.E. Cates et al, J. Phys. Cond. Matt, 16, S3903 (2004).

[27] J.C. Meiners and S.R. Quake, Phys. Rev. Lett 82, 2211 (1999).

[28] A.J.C. Ladd, private communication, obtained similar results to those shown for his algorithm in figure 3 after reading an earlier draft of this paper in which we discussed only the case of $N>1$.

[29] For our recommended range of $k_{B} T \leq 1 / 3000$, colloidal equilibration remains good to two or three percent. At much higher temperatures, ERs significantly larger than unity arise in the fluid and (for uncertain reasons) are amplified further in the colloid sector.

[30] We quote standard errors of the mean but note that the statistical error might be larger in some cases due to long-lived hydrodynamic correlations; we find these to be weak for our parameters. 